\title{
Physical Inactivity Is Correlated with Levels of Quantitative C-reactive Protein in Serum, Independent of Obesity: Results of the National Surveillance of Risk Factors of Non-communicable Diseases in Iran
}

\author{
Alireza Esteghamati, Afsaneh Morteza, Omid Khalilzadeh, Mehdi Anvari, \\ Sina Noshad, Ali Zandieh, and Manouchehr Nakhjavani \\ Endocrinology and Metabolism Research Center, Vali-Asr Hospital, \\ School of Medicine, Tehran University of Medical Sciences, Tehran, Iran
}

\begin{abstract}
Increased C-reactive protein (CRP) levels are associated with coronary heart disease, stroke, and mortality. Physical activity prevents cardiovascular disorders, which can be partly mediated through reducing inflammation, including serum CRP levels. The association of different intensities of physical activity, sedentary behaviours, and C-reactive protein (CRP) levels in serum was examined after adjustment for markers of adiposity, including waist-circumference and body mass index (BMI), in a large population-based study. Using data of the SuRFNCD-2007 study, a large national representative population-based study in Iran, the relationship between quantitative CRP concentrations in serum and physical activity was examined in a sample of 3,001 Iranian adults. The global physical activity questionnaire (GPAQ) was used for evaluating the duration and intensity of physical activity. Total physical activity (TPA) was calculated using metabolic equivalents for the intensity of physical activity. Quantitative CRP concentrations in serum were measured with high-sensitivity enzyme immunoassay. The CRP levels in serum significantly correlated with TPA ( $\mathrm{r}=-0.103, \mathrm{p}=0.021$ in men and $\mathrm{r}=-0.114, \mathrm{p}=0.017$ in women), duration of vigorous-intensity activity $(\mathrm{r}=-0.122, \mathrm{p}=0.019$ in men and $\mathrm{r}=-0.109, \mathrm{p}=0.026$ in women), duration of moderate-intensity activity $(\mathrm{r}=-$ 0.107, $\mathrm{p}=0.031$ in men and $\mathrm{r}=-0.118, \mathrm{p}=0.020$ in women), and duration of sedentary behaviours ( $\mathrm{r}=0.092$, $\mathrm{p}=0.029$ in men and $\mathrm{r}=0.101, \mathrm{p}=0.022$ in women) after multiple adjustments for age, area of residence, BMI, waist-circumference, smoking, and diabetes mellitus. Physical activity (of both moderate and vigorous intensity) is inversely associated with the quantitative CRP levels in serum, independent of diabetes and body adiposity.
\end{abstract}

Key words: Cardiovascular diseases; C-reactive protein; Physical activity; Physical exercise; Risk factors; Iran

\section{INTRODUCTION}

Adipose tissue in obesity, diabetes, and metabolic syndrome is in a state of chronic inflammation (1-5). Quantitative C-reactive protein (CRP), an acute-phase reactant produced dominantly by liv-

Correspondence and reprint requests should be addressed to:

Dr. Alireza Esteghamati

Professor of Endocrinology and Metabolism Endocrinology and Metabolism Research Center

Vali-Asr Hospital, School of Medicine

Tehran University of Medical Sciences

PO Box 13145-784, Tehran

Iran

Email: esteghamati@tums.ac.ir

Fax: +982164432466 er, is a plasma protein that circulates in increased amounts during inflammation and often damages the tissue $(6,7)$. Results of studies showed that the increased CRP levels were associated with functional impairment, coronary heart disease, stroke, and mortality (8). So, reducing the CRP levels can reduce mortality and morbidity due to cardiovascular disorders.

Physical activity can prevent cardiovascular disorders (9). This effect can be partly mediated through reducing inflammation, including serum CRP levels (10). Results of some population-based studies showed a negative association between physical activity and the circulating levels of CRP $(7,10-14)$. However, the pattern of this association with respect to different intensities and metabolic equiva- 
lents (METs) of physical activity and several tertiary factors, such as adiposity and glycaemic status, is not well-understood. Results of some studies showed that the impact of physical activity is mediated through its weight-lowering effect $(15,16)$. A study reported that the circulating levels of CRP can be markedly suppressed, independent of total adiposity or fat mass, by intense regular physical exercise (17).

The present study was carried out to examine the association among different intensities of physical activity (moderate and severe), total physical activity (using METs for the intensity of activities), duration of sedentary behaviours, and the serum CRP levels after adjustment for glycaemic status and markers of adiposity, including waist-circumference and body mass index (BMI) in a large populationbased sample of Iranian adults. Physical activity was defined by an international standard questionnaire, named global physical activity questionnaire (GPAQ).

\section{MATERIALS AND METHODS}

\section{Participants}

The study was based on data collected in the third national surveillance of risk factors of non-communicable diseases (SuRFNCD-2007). Details of the survey were reported elsewhere (18). In brief, a cluster-sampling scheme was applied to randomly select a representative sample of Iranian adults aged 25-64 years. The number of clusters selected from each province was proportional to the urban/rural size of that province. Trained healthcare professionals conducted household interviews and physical examinations. All interviews were conducted in Persian. Data were recorded in standardized sets of questionnaire. Blood sampling was done within a few days of the interview.

The survey received ethical approval from the Center for Disease Control of Iran, and written informed consent was obtained from all the participants.

\section{Assessment of physical activity}

The second version of the GPAQ was used in the survey (19). This questionnaire, developed by the World Health Organization (WHO), contains 16 questions about physical activity in a typical week and assesses physical activity in three domains: work, transportation, and recreational activities. The evaluation of physical activity in these domains is one of the factors that make the GPAQ distinct from other sets of questionnaire, such as the less-sophisticated, short version of the international physical activity questionnaire (IPAQ) (http:// www.ipaq.ki.se). It also determines the intensity of activity (i.e. vigorous or moderate) in each domain and the time spent on sedentary behaviours, such as watching TV. Sedentary behaviours were defined as activities, such as sitting at a desk, travelling in car/bus/train, reading, working with computer, and watching television.

To measure energy expenditure, the concept of METs was used (19). MET is the ratio of a person's working metabolic rate and the resting metabolic rate (19). One MET is defined as the energy cost of sitting quietly and is equivalent to a caloric consumption of $1 \mathrm{kcal} / \mathrm{kg} /$ hour. It is estimated that a person's caloric consumption is four times high when moderately active and eight times high when vigorously active. Therefore, to calculate a person's overall energy expenditure, four METs are assigned to the time spent on moderate activities, and eight METs are assigned to the time spent on vigorous activities. The total physical activity (TPA) score was calculated as the sum of all METs $\times$ minutes for moderate- or vigorous-intensity physical activity performed in work, commuting, and recreation.

Based on the GPAQ analysis framework (19), our participants were classified into three groups of high, moderate and low physical activity categories. More details on these definitions were reported elsewhere $(20,21)$.

\section{Physical examination and biochemical measurements}

Weight and height were measured in light clothing and without shoes. A portable calibrated electronic weighing scale (Omron Corp., Tokyo, Japan) and portable measuring inflexible bars (Seca, Hamburg, Germany) were used for this purpose. A constant tension tape (Seca, Hamburg, Germany) was used for measuring waist-circumference at the end of a normal expiration, with arms relaxed at the sides, at the midpoint between the lower margin of the lowest rib and the highest point of the hip on the mid-axillary line. The BMI $\left(\mathrm{kg} / \mathrm{m}^{2}\right)$ was calculated according to the Quetelet formula. Five $\mathrm{mL}$ of venous blood was taken in sitting position, centrifuged, and transferred under cold-chain condition to the laboratory. Quantitative CRP concentrations in serum were measured in the endocrine laboratory of the Vali-Asr Hospital (Tehran University of Medical Sciences) using the quantitative CRP kit (Parsazmoon, Karaj, Iran), with an intra-assay coefficient of variation of 2.6\%. Diabetes was diagnosed following the criteria of the American Diabetes Association as described in our previous report (22). 


\section{Statistical analysis}

Complex analysis of the sample survey was performed using the SPSS software for Windows (version 17) (Chicago, IL, USA). Data were weighted for sex, age, and residential area (urban/rural) strata, according to the population of Iran (national census, 2006). Kolmogorov Smirnov analysis was employed to study the normality of variables. CRP concentrations in serum were log-transformed to change the distribution to normal; the log-transformed values of CRP were used in analyses. Continuous variables were expressed as mean \pm standard error of mean (SEM). Partial correlation coefficients were calculated between quantitative CRP and features of physical activity after adjustment for various variables. The adjusted values of quantitative CRP were compared between the categories of physical activity, using the general linear modelling method. The $p$ value of $<0.05$ was considered significant.

\section{RESULTS}

After excluding the participants with missing data in laboratory results $(\mathrm{n}=396)$, analyses were performed for the remaining 3,001 individuals. The demographic data (age, sex, and residential area) of the excluded subjects were not different from the remaining participants. Table 1 shows the baseline characteristics of the study participants. Forty-six percent of men were in the category of high physi- cal activity while $40 \%$ of women fell into the category of low physical activity. There was no significant difference in the serum CRP levels between men and women.

The serum CRP levels correlated with age $(\mathrm{r}=0.058$, $\mathrm{p}=0.024$ in men and $\mathrm{r}=0.065$, $\mathrm{p}=0.44$ in women), BMI ( $\mathrm{r}=0.029, \mathrm{p}=0.026$ in men and $\mathrm{r}=0.030, \mathrm{p}=0.023$ in women), and waist-circumference ( $\mathrm{r}=0.59$, $\mathrm{p}=0.021$ in men and $\mathrm{r}=0.047, \mathrm{p}=0.046$ in women). Current smokers had higher levels of quantitative CRP vs ex-smokers or never-smokers $(5.64 \pm 3.06$ vs $5.19 \pm 2.73, \mathrm{p}=0.139$ in men and $5.60 \pm 2.68$ vs $5.41 \pm 2.93, \mathrm{p}=0.69$ in women). TPA was inversely associated with age $(\mathrm{r}=-0.212, \mathrm{p}=\mathrm{p} .002$ in men and $\mathrm{r}=-0.173, \mathrm{p}=0.005$ in women), BMI ( $\mathrm{r}=-0.338$, $\mathrm{p}<0.001$ in men and $\mathrm{r}=-0.198, \mathrm{p}=0.001$ in women), and waist-circumference ( $r=-0.357, p<0.001$ in men and $\mathrm{r}=-0.239, \mathrm{p}<0.001$ in women).

CRP significantly correlated with TPA ( $\mathrm{r}=-0.103$, $\mathrm{p}=0.021$ in men and $\mathrm{r}=-0.114, \mathrm{p}=0.017$ in women), duration of vigorous-intensity activity ( $\mathrm{r}=-$ $0.122, \mathrm{p}=0.019$ in men and $\mathrm{r}=-0.109, \mathrm{p}=0.026$ in women), duration of moderate-intensity activity $(\mathrm{r}=-0.107, \mathrm{p}=0.031$ in men and $\mathrm{r}=-0.118, \mathrm{p}=0.020$ in women), and duration of sedentary behaviours $(\mathrm{r}=0.092, \mathrm{p}=0.029$ in men and $\mathrm{r}=0.101, \mathrm{p}=0.022$ in women) after multiple adjustments for age, area of residence, BMI, waist-circumference, smoking, and diabetes mellitus using partial correlation (Table 2).

\begin{tabular}{|c|c|c|c|}
\hline Characteristics & $\begin{array}{c}\text { Male } \\
(\mathrm{n}=1,494)\end{array}$ & $\begin{array}{c}\text { Female } \\
(\mathrm{n}=1,507)\end{array}$ & $\begin{array}{c}\text { Total } \\
(\mathrm{n}=3,001)\end{array}$ \\
\hline Age (year) ${ }^{*}$ & $39.42 \pm 0.75$ & $39.76 \pm 0.78$ & $39.59 \pm 0.54$ \\
\hline \multicolumn{4}{|l|}{ Area of residence } \\
\hline Urban (\%) & $1,011(67.7)$ & $1,004(66.6)$ & $2,015(67.1)$ \\
\hline Rural (\%) & $483(32.3)$ & $503(33.4)$ & $986(32.9)$ \\
\hline $\mathrm{BMI}\left(\mathrm{kg} / \mathrm{m}^{2}\right)^{*}$ & $25.4 \pm 0.2$ & $27.6 \pm 0.2$ & $26.5 \pm 0.2$ \\
\hline Waist-circumference $(\mathrm{cm})^{*}$ & $88.6 \pm 0.4$ & $88.8 \pm 0.6$ & $88.7 \pm 0.4$ \\
\hline \multicolumn{4}{|l|}{ Physical activity category } \\
\hline Low $(\%)$ & $31.6 \pm 1.1$ & $48.6 \pm 1.3$ & $40.0 \pm 1.0$ \\
\hline Moderate (\%) & $22.3 \pm 0.1$ & $27.1 \pm 1.2$ & $24.7 \pm 0.8$ \\
\hline High (\%) & $46.1 \pm 1.3$ & $24.3 \pm 1.2$ & $35.4 \pm 1.1$ \\
\hline Total physical activity (MET-min/day) & $909.2 \pm 47.3$ & $327.9 \pm 13.8$ & $623.2 \pm 31.9$ \\
\hline Duration of vigorous activity (min/day) & $58.1 \pm 5.7$ & $4.9 \pm 0.9$ & $31.9 \pm 3.9$ \\
\hline Duration of moderate activity (min/day) & $111.1 \pm 4.4$ & $72.2 \pm 2.3$ & $91.9 \pm 3.2$ \\
\hline Duration of sedentary behaviours (min/day) & $240.2 \pm 4.2$ & $238.0 \pm 4.6$ & $239.2 \pm 3.8$ \\
\hline Current smoking $(\% \pm \mathrm{SE})$ & $26.1 \pm 1.6$ & $1.8 \pm 0.3$ & $14.1 \pm 1.1$ \\
\hline Diabetes mellitus & $6.6 \pm 0.4$ & $9.1 \pm 0.4$ & $7.8 \pm 0.4$ \\
\hline Quantitative CRP $(\mathrm{mg} / \mathrm{L})^{*}$ & $5.6 \pm 0.1$ & $5.6 \pm 0.1$ & $5.6 \pm 0.1$ \\
\hline
\end{tabular}




\begin{tabular}{|c|c|c|c|c|}
\hline \multirow{2}{*}{ Total physical activity (MET-min/day) } & \multicolumn{2}{|c|}{ Male } & \multicolumn{2}{|c|}{ Female } \\
\hline & $\mathrm{r}$ & $\mathrm{p}$ & $\mathrm{r}$ & $\mathrm{p}$ \\
\hline \multicolumn{5}{|l|}{ Adjustment for } \\
\hline Age and area of residence & -0.127 & 0.011 & -0.129 & 0.010 \\
\hline Age, area of residence, BMI, WC, and smoking & -0.103 & 0.021 & -0.114 & 0.017 \\
\hline $\begin{array}{l}\text { Age, area of residence, BMI, WC, } \\
\text { smoking, diabetes mellitus, and } \\
\text { duration of sedentary behaviours }\end{array}$ & -0.084 & 0.035 & -0.104 & 0.030 \\
\hline \multicolumn{5}{|l|}{ Duration of vigorous activity (min/day) } \\
\hline \multicolumn{5}{|l|}{ Adjustment for } \\
\hline Age and area of residence & -0.131 & 0.008 & -0.117 & 0.021 \\
\hline Age, area of residence, BMI, WC, and smoking & -0.122 & 0.019 & -0.109 & 0.026 \\
\hline $\begin{array}{l}\text { Age, area of residence, BMI, WC, } \\
\text { smoking, diabetes mellitus, duration } \\
\text { of moderate activity, and sedentary } \\
\text { behaviours }\end{array}$ & -0.116 & 0.027 & -0.106 & 0.029 \\
\hline \multicolumn{5}{|l|}{ Duration of moderate activity (min/day) } \\
\hline \multicolumn{5}{|l|}{ Adjustment for } \\
\hline Age and area of residence & -0.123 & 0.018 & -0.125 & 0.012 \\
\hline Age, area of residence, $\mathrm{BMI}, \mathrm{WC}$, and smoking & -0.107 & 0.031 & -0.118 & 0.020 \\
\hline $\begin{array}{l}\text { Age, area of residence, BMI, WC, } \\
\text { smoking, diabetes mellitus, duration } \\
\text { of vigorous activity, and sedentary } \\
\text { behaviours }\end{array}$ & -0.071 & 0.052 & -0.102 & 0.032 \\
\hline \multicolumn{5}{|l|}{ Duration of sedentary behaviours (min/day) } \\
\hline \multicolumn{5}{|l|}{ Adjustment for } \\
\hline Age and area of residence & 0.108 & 0.013 & 0.119 & 0.008 \\
\hline Age, area of residence, BMI, WC, and smoking & 0.092 & 0.029 & 0.101 & 0.022 \\
\hline $\begin{array}{l}\text { Age, area of residence, BMI, WC, } \\
\text { smoking, diabetes mellitus, and TPA }\end{array}$ & 0.081 & 0.041 & 0.078 & 0.047 \\
\hline
\end{tabular}

The correlation between quantitative CRP and TPA was independent of the duration of sedentary behaviours ( $\mathrm{r}=-0.084, \mathrm{p}=0.035$ in men and $\mathrm{r}=-0.104$, $\mathrm{p}=0.030$ in women) (Table 2).

Considering the different categories of physical activity, there was a significant inverse correlation between quantitative CRP and higher physical activity in both men and women after multiple adjustments for age, residential area, BMI, waist-circumference, smoking, and diabetes mellitus (Fig.).

Serum CRP concentrations of $\geq 10 \mathrm{mg} / \mathrm{L}$ are frequently observed in subjects with inflammatory conditions (23); therefore, in a separate analysis, we excluded these patients $(n=70)$ and repeated the association analyses once more. This change could not considerably change the level of associations (r) in different models.

\section{DISCUSSION}

In this study, the duration and the intensity (vigorous and moderate) of physical activity in three domains of work, transportation, and leisure time were evaluated using the GPAQ. Our findings clearly demonstrated that physical activities of both moderate and vigorous intensity were associated with lower levels of quantitative CRP. Furthermore, we showed that this correlation was independent of several potential confounders, including BMI, waist-circumference, smoking status, diabetes mellitus, age, and sex. Note that our study, in a large population-based sample, could show a mild but significant and independent inverse association between physical activity and CRP. The mild degree of this association is expected because, in a general population, the level of CRP does not have a large variation, and the majority of people have normal 


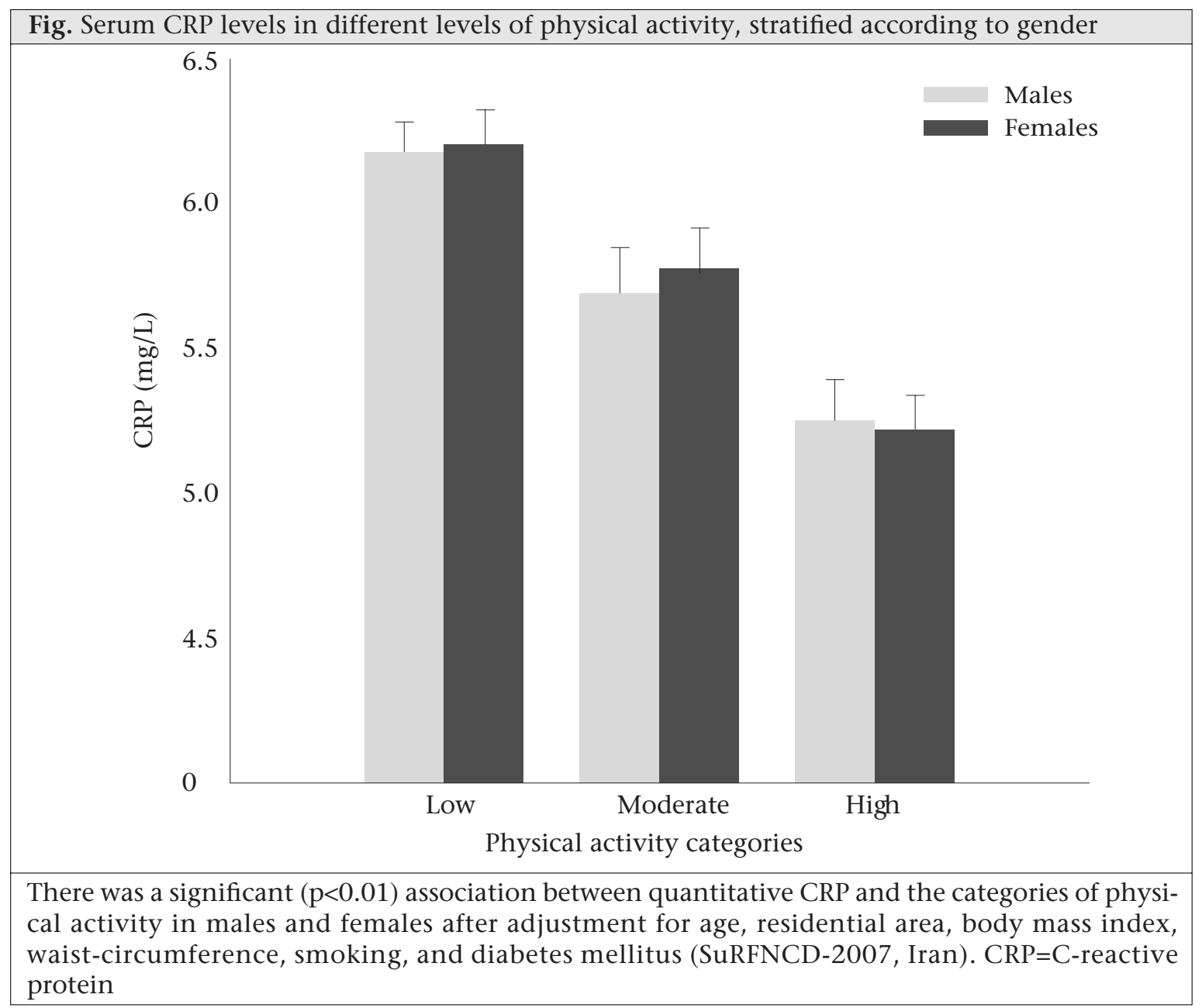

ranges of CRP. However, our findings are important because it shows that, even in a general sample of a population where most people have a normal range of CRP, the level of physical activity is an important indicator, which contributes to the mild differences of CRP levels among different individuals of the population.

Our results are generally consistent with those of the growing number of studies, suggesting that high levels of physical activity are associated with lower levels of quantitative CRP $(7,10)$. In a study which evaluated the effect of physical activity on serum CRP levels among 3,075 men and women, higher levels of exercise were associated with lower levels of quantitative CRP (11). In a cohort of 5,888 men and women aged over 65 years, those in the highest quartile of physical activity had 19\% lower concentrations of quantitative CRP compared to persons in the lowest quartile (12). In another study, physical activity was significantly and inversely associated with several inflammatory variables, including quantitative CRP (13). They also showed that, after a 20-year follow- up, the quantitative CRP levels of those who were inactive at first but later took up light physical activity reached the CRP levels of the participants who were active throughout the follow-up (13). Physical activity was independently associated with lower odds of having an elevated level of quantitative CRP in the Third National Health and Nutrition Examination Survey (14). Compared to these studies, our study has the advantage of evaluating the impact of different intensities of physical activity using the concept of METs. Furthermore, our study could show that this association was independent of indicators of body adiposity and diabetes. Our findings also, for the first time, point to a significant association between the time spent on sedentary behaviour and the quantitative CRP levels, independent of physical activity and obesity.

Consistent with our findings, Fischer and colleagues showed that the plasma levels of CRP were associated with physical inactivity, independent of obesity (24). Similarly, Tomaszewski and colleagues showed that the circulating levels of CRP could be markedly suppressed, independent of total adiposity or indeed fat mass, by intense regular 
physical exercise (17). However, they had a small sample-size to conclude this finding. Why does physical activity reduce the serum CRP levels? Various theories have been postulated to explain this (25-27). The serum levels of TNF- $\alpha$ and IL-6 were reduced by physical activity, independent of the adipose tissue content (27). TNF- $\alpha$ and IL- 6 induce the production and secretion of CRP (28). So, this may reduce the serum CRP levels, irrespective of fat content. On the other hand, physical activity induces anti-inflammatory cytokines, including IL-1 receptor antagonist and IL-10, which are known to hamper the production of CRP $(26,27)$. We did not measure the levels of IL- 6 and TNF- $\alpha$ in serum in our participants. Nevertheless, we encourage other researchers to evaluate the serum level of different cytokines in parallel with CRP and physical activity in the future epidemiologic studies.

Previous studies gave different weights to the health effects of moderate and vigorous physical activity (29). Some authors suggest that vigorous activity is much stronger in improving cardiovascular health but others argue that moderate activity (which can be tolerated longer in an ordinary man) can be as effective as vigorous activity (30). Our results showed that activities of both vigorous and moderate intensity were independently associated with the lower CRP level but the degree of association was higher for vigorous vs moderate in both males and females. Therefore, our study provides a support for the beneficial consequences of activities of both vigorous and moderate intensity.

\section{Limitations}

The principal limitation of the present study is its cross-sectional nature, which precludes the determination of the direction of causality. This study was conducted in accordance with the stepwise guidelines of $\mathrm{WHO}$, and our questionnaire did not gather data on history of cardiovascular diseases, malignancy, or other acute/chronic diseases (22). The decision regarding non-inclusion of history of cardiovascular diseases in the SuRFNCD questionnaire comes from the fact that there is no readilyavailable technique to diagnose these conditions in the cases of negative history. For example, doing exercise testing or angiography on a national scale is not feasible. Therefore, the influence of these factors was not assessed in our study; however, we believe that this limitation does not lessen the importance of our findings to show a significant crude association between the CRP levels and physical inactivity in a general population. Also, factors, such as diet or alcohol drinking status, may also be influential. Nevertheless, drinking of alcohol is illegal in Iran, and based on our experience, a very low percentage of our population was drinkers.
The principal advantage of the present study is its large sample-size, and the point that it is representative of the Iranian population. Moreover, we used a standardized international questionnaire to study the different domains of physical activity and sedentary behaviours. We also evaluated the potential confounding effects of several tertiary factors on the association between physical activity and quantitative CRP.

\section{Conclusions}

In this study, we evaluated the association between different intensities of physical activity and the serum CRP level. We showed that both vigorous and moderate physical activities were associated with the lower serum CRP levels. This association was independent of diabetes and body adiposity. We also showed that the time spent in sedentary behaviours positively correlated with the quantitative CRP levels, independent of physical activity and obesity. The practical message of the study is that any type of physical activity (vigorous or moderate) is associated with lower CRP and can, therefore, be beneficial for a healthier lifestyle. Low activity and sedentary behaviours are independently associated with high CRP and can potentially cause negative health consequences.

\section{ACKNOWLEDGEMENTS}

The study was supported by the Ministry of Health and Medical Education, Tehran, Iran.

\section{REFERENCES}

1. Fernandez-Real JM, Vayreda M, Richart C, Gutierrez $\mathrm{C}$, Broch M, Vendrell J et al. Circulating interleukin 6 levels, blood pressure, and insulin sensitivity in apparently healthy men and women. J Clin Endocrinol Metab 2001;86:1154-9.

2. Tracy RP, Lemaitre RN, Psaty BM, Ives DG, Evans RW, Cushman $\mathrm{M}$ et al. Relationship of C-reactive protein to risk of cardiovascular disease in the elderly. Results from the Cardiovascular Health Study and the Rural Health Promotion Project. Arterioscler Thromb Vasc Biol 1997;17:1121-7.

3. Ridker PM, Hennekens CH, Buring JE, Rifai N. Creactive protein and other markers of inflammation in the prediction of cardiovascular disease in women. N Engl J Med 2000;342:836-43.

4. Ridker PM, Rifai N, Stampfer MJ, Hennekens CH. Plasma concentration of interleukin- 6 and the risk of future myocardial infarction among apparently healthy men. Circulation 2000;101:1767-72.

5. Fröhlich M, Imhof A, Berg G, Hutchinson WL, Pepys MB, Boeing $\mathrm{H}$ et al. Association between C-reactive protein and features of the metabolic syndrome: a population-based study. Diabetes Care 2000;23:1835-9. 
6. Opal SM, DePalo VA. Anti-inflammatory cytokines. Chest 2000;117:1162-72.

7. Yu Z, Ye X, Wang J, Qi Q, Franco OH, Rennie KL et al. Associations of physical activity with inflammatory factors, adipocytokines, and metabolic syndrome in middle-aged and older chinese people. Circulation 2009;119:2969-77.

8. Emerging Risk Factors Collaboration, Kaptoge S, Di Angelantonio E, Lowe G, Pepys MB, Thompson SG et al. C-reactive protein concentration and risk of coronary heart disease, stroke, and mortality: an individual participant meta-analysis. Lancet 2010;375:132-40.

9. Thompson PD, Buchner D, Pina IL, Balady GJ, Williams MA, Marcus BH et al. Exercise and physical activity in the prevention and treatment of atherosclerotic cardiovascular disease: a statement from the Council on Clinical Cardiology (Subcommittee on Exercise, Rehabilitation, and Prevention) and the Council on Nutrition, Physical Activity, and Metabolism (Subcommittee on Physical Activity). Circulation 2003;107:3109-16.

10. Ford ES. Does exercise reduce inflammation? Physical activity and C-reactive protein among U.S. adults. Epidemiology 2002;13:561-8.

11. Colbert LH, Visser M, Simonsick EM, Tracy RP, Newman AB, Kritchevsky SB et al. Physical activity, exercise, and inflammatory markers in older adults: findings from the Health, Aging and Body Composition Study. J Am Geriatr Soc 2004;52:1098-104.

12. Geffken DF, Cushman M, Burke GL, Polak JF, Sakkinen PA, Tracy RP. Association between physical activity and markers of inflammation in a healthy elderly population. Am J Epidemiol 2001;153:242-50.

13. Wannamethee SG, Lowe GD, Whincup PH, Rumley A, Walker M, Lennon L. Physical activity and hemostatic and inflammatory variables in elderly men. Circulation 2002;105:1785-90.

14. Abramson JL, Vaccarino V. Relationship between physical activity and inflammation among apparently healthy middle-aged and older US adults. Arch Intern Med 2002;162:1286-92.

15. Miller GD, Nicklas BJ, Loeser RF. Inflammatory biomarkers and physical function in older, obese adults with knee pain and self-reported osteoarthritis after intensive weight-loss therapy. J Am Geriatr Soc 2008;56:644-51.

16. Moran LJ, Noakes M, Clifton PM, Wittert GA, Belobrajdic DP, Norman RJ. C-reactive protein before and after weight loss in overweight women with and without polycystic ovary syndrome. J Clin Endocrinol Metab 2007;92:2944-51.

17. Tomaszewski M, Charchar FJ, Przybycin M, Crawford L, Wallace AM, Gosek K et al. Strikingly low circulating CRP concentrations in ultramarathon runners independent of markers of adiposity: how low can you go? Arterioscler Thromb Vasc Biol 2003;23:1640-4.

18. Esteghamati A, Meysamie A, Khalilzadeh O, Rashidi A, Haghazali M, Asgari F et al. Third National Surveillance of Risk Factors of Non-Communicable Diseases (SuRFNCD-2007) in Iran: methods and results on prevalence of diabetes, hypertension, obesity, central obesity, and dyslipidemia. BMC Public Health 2009;9:167.

19. World Health Organization. Global physical activity questionnaire (GPAQ): analysis guide. Geneva: World Health Organization. 23 p. (http://www.who. int/chp/steps/resources/GPAQ_Analysis_Guide.pdf, accessed on 1 November 2009).

20. Esteghamati A, Khalilzadeh O, Ashraf H, Zandieh A, Morteza A, Rashidi A et al. Physical activity is correlated with serum leptin independent of obesity: results of the national surveillance of risk factors of noncommunicable diseases in Iran (SuRFNCD-2007). Metabolism 2010;59:1730-5.

21. Esteghamati A, Khalilzadeh O, Rashidi A, Meysamie A, Haghazali M, Asgari F et al. Association between physical activity and insulin resistance in Iranian adults: National Surveillance of Risk Factors of NonCommunicable Diseases (SuRFNCD-2007). Prev Med 2009;49:402-6.

22. Esteghamati A, Zandieh A, Khalilzadeh O, Meysamie A, Ashraf H. Clustering of metabolic syndrome components in a Middle Eastern diabetic and non-diabetic population. Diabetol Metab Syndr 2010;2:36.

23. Pepys MB, Hirschfield GM. C-reactive protein: a critical update. J Clin Invest 2003;111:1805-12.

24. Fischer CP, Berntsen A, Perstrup LB, Eskildsen P, Pedersen BK. Plasma levels of interleukin-6 and Creactive protein are associated with physical inactivity independent of obesity. Scand J Med Sci Sports 2007;17:580-7.

25. Kasapis C, Thompson PD. The effects of physical activity on serum C-reactive protein and inflammatory markers: a systematic review. J Am Coll Cardiol 2005;45:1563-69.

26. Petersen AM, Pedersen BK. The role of IL-6 in mediating the anti-inflammatory effects of exercise. J Physiol Pharmacol 2006;57(Suppl 10):43-51.

27. Jung SH, Park HS, Kim KS, Choi WH, Ahn CW, Kim BT et al. Effect of weight loss on some serum cytokines in human obesity: increase in IL-10 after weight loss. J Nutr Biochem 2008;19:371-5.

28. Boncler M, Watala C. Regulation of cell function by isoforms of C-reactive protein: a comparative analysis. Acta Biochim Pol 2009;56:17-31.

29. Swain DP, Franklin BA. Comparison of cardioprotective benefits of vigorous versus moderate intensity aerobic exercise. Am J Cardiol 2006;97:141-7.

30. Haennel RG, Lemire F. Physical activity to prevent cardiovascular disease. How much is enough? Can Fam Physician 2002;48:65-71. 